\title{
Stridences et chuchotements : la symphonie des machines et des portes au XIX ${ }^{\mathrm{e}}$ siècle
}

Le citadin de la rue au XIX siècle, peu ou pas fortuné, vit dans des atmosphères sonores. Le bourgeois tente, lui, de s'en fabriquer. Cet épisode historique atteste deux attitudes différentes dans la ville, de la part d'individus qui occupent pourtant le même espace sensible, attitudes qui, semble-t-il, entrent en résonance avec des formes d'engagement rencontrées aujourd'hui.

Pour en témoigner, deux écrivains, Zola et Balzac, seront nos guides. Leurs propos pourront paraître caricaturaux mais ils sont d'une extrême efficacité pour évoquer les deux sensibilités phoniques d'alors. L'univers sonore de Zola plonge d'emblée le lecteur dans le tumulte et l'agitation d'un monde que l'industrialisation et l'urbanisation ont profondément modifié au cours du XIX ${ }^{\mathrm{e}}$ siècle ; les ambiances phoniques décrites dans les ouvrages de Balzac font prendre conscience d'un univers précautionneux et porté par des personnages curieux d'entendre les dimensions acoustiques de la ville les plus intimes.

Il n'y a pas de véritable politique du bruit à cette époque. Les convictions bourgeoises sont source d'une longue suite d'interdits dans le cadre domestique et l'espace public disant quelles sont les règles de comportement sonore les plus appropriées. Le bourgeois cherche à rendre la présence sonore des domestiques autour de lui moralement acceptable, à styliser les gestes et les intonations, à définir des cadres temporels où les bruits, quand la nécessité du service s’impose, sont compatibles avec des « techniques sur soi » propres à la morale de vie. Pour les plus démunis, face au bruit qui s'intensifie et qui devient de plus en plus continu, la "déviance individuelle », comme le dit Yves Lequin ${ }^{1}$, est une manière de répondre à une situation et à une déculturation urbaine nouvelles ${ }^{2}$. Mais ces déviances les plus audibles sont l'objet d'une incompréhension à peu près totale, et donc de censures. De la méfiance on glisse à la peur et aux fausses convictions. Ainsi, les sources d'archives ${ }^{3}$ permettent de saisir les réactions d'une 


\section{Olivier Balä̈}

autorité tracassée par le vacarme d'une vogue, par le bruit des hommes qui nettoient les fosses d'aisances, par les crieurs publics. Or ces incompréhensions et peurs bourgeoises apparaissent au moment où l'installation et le fonctionnement en ville d'une industrie bruyante sont l'objet d'une grande indulgence.

\section{LES RYTHMES ET LA VIVACITÉ DES SONS : ZOLA}

La preuve de cette indulgence? On la trouve dans les premières lignes de L'Assommoir ${ }^{4}$. Le monde sonore de Gervaise se définit entre le grondement de la ville au travail et les contacts qui s'engagent de fenêtre à rue ou de fenêtre à fenêtre avec les voisins. L'écriture de Zola raconte l'interaction de l'écoute et de la motricité. On lit le peuple des ouvriers en déplacement qui « gronde», «s'arrête», « repart»; cela ressemble au bruit mouvant des vagues. Ailleurs, les personnages sont interpellés depuis la rue, et aussitôt prend forme une discussion qui à son tour en appelle d'autres. Zola nous conduit ainsi à travers L'Assommoir et Au Bonheur des Dames à la situation du citadin interagissant avec les sons qui l'entourent. Les bruits agissent sur les actes, sur les pensées, ils circulent entre les personnages et «organisent» la motricité, celle d'une foule ou celle d'un individu. Prenons, pour nous en rendre compte, cinq textes événementiels : un repas lors d'une fête dans la rue, une scène de travail à l'usine, une scène de travail chez l'artisan, un bain de foule sur les grands boulevards et la description des conditions de travail dans les grands magasins.

\section{La fête de Gervaise.}

Durant tout le chapitre ? de L'Assommoir, il n'est question que de gens qui parlent fort, crient, chantent, gueulent, s'injurient, s'engueulent, font du vacarme, avec leur voix et avec leur corps. Un rythme sonore renforce ce sentiment dans une sorte d'accélération. Les sons adviennent et sont produits de plus en plus rapidement et de plus en plus intensément. Il y a des ruptures: une voix forte impose le silence, un profond silence tout d'un coup se fait. Ensuite, l'ambiance phonique va s'accélérant selon les propres rythmes et gestes sonores des participants : les hommes marquent la mesure à coups de talon, les femmes en tapant en cadence sur les verres, les enfants avec leurs fourchettes. Enfin, il y a le rythme des sensations sonores collectives : la «communion» qui s'établit par moment avec 
la rue, les applaudissements qui font monter l'ambiance, la chanson de Mme Putois qui la coupe, tout cela organise un temps d'écoute collectif et redonne des élans à la fête. Puis c'est de nouveau un attroupement dans la rue, la rue de la Goutte-d'Or, qui participe au refrain. La fête déborde et, de façon inattendue, les gens «s'allongent des claques pour rire » en chantant.

\section{Scènes de travail à l'usine et à l'atelier.}

Dans les nouveaux lieux technologiques de la ville, c'est ce même enchâ̂nement qui guide l'écrivain décrivant d'autres inductions sonores en chaîne. Toute la fin de L'Assommoir l'illustre. Comme Gervaise déambule affamée dans Paris, elle se retrouve dans un quartier où vient " de s'établir une scierie de sucre dont on [entend] les sifflements continus ${ }^{5} »$. Zola décrit: Gervaise "entendait dans ce vaste espace clair des sifflets de locomotives, les secousses rythmées des plaques tournantes, toute une activité colossale et cachée. Puis un train passa, sortant de Paris, arrivant avec les soufflements de son haleine et son roulement peu à peu enflé ${ }^{6}$ ». C'est alors au milieu du son aigu du tapage des usines qu'elle s'oriente :

De minces tuyaux sur les toits soufflaient violemment des jets de vapeur. Une scierie mécanique avait des grincements réguliers pareils à de brusques déchirures dans une pièce de calicot; des manufactures de boutons secouaient le sol du roulement et du tic tac de leurs machines. Comme elle regardait vers Montmartre, indécise, ne sachant pas si elle devait pousser plus loin [...], elle entendit un bruit cadencé de marteaux : elle était sans le savoir juste en face de la fabrique $[\ldots]$. Le bruit des marteaux avait cessé. Elle s'avançait prudemment. [Elle demanda à voir Goujet] un ouvrier lui répondit en criant d'une voix qui sonnait le cuivre fêlé : «Dis donc, la Gueule d'or, voilà une dame pour toi. » Mais un tapage de ferraille étouffa ce cri. [...] Gervaise, n'osant s'aventurer, appelait de la porte à demi-voix $[\ldots]$. Elle $[\ldots]$ se posa devant lui. [... Il marcha le premier, elle le suivit, dans ce vacarme assourdissant où toutes sortes de bruits sifflaient et ronflaient $[\ldots]$. On ne s'entendait pas parler $[\ldots]$. Il haussa la voix pour donner des explications?

À l'usine, le rythme installé est de l'ordre de l'emballement; on y perd la tête, le corps est désorienté ; le bruit remplit tout l'espace et l'ouvrier se sent comme prisonnier. La description sonore de l'atelier de repassage de Gervaise a une tout autre valeur: c'est «au milieu des bruits de fer et de tisonniers grattant la mécanique » que le ronflement de Coupeau roule, «avec la régularité d'un tic tac énorme d'horloge, régulant la grosse 


\section{Olivier Balä̈}

besogne de l'atelier» de repassage. On n'entend «que les coups sourds, étouffés sur la couverture [et] une voix lente et continue [qui], sans quitter des yeux le petit fer à tuyauter», parle «dans le silence». Quand le « grand sommeil » tombe lentement sur la ville le soir, c'est encore le rythme de l'horloge qui accompagne le travail: «Minuit sonnait, puis une heure, puis deux heures »; il n'y a en effet plus de voitures ni de passants, sauf de temps en temps un pas qui sonne au loin ${ }^{8}$. À l'atelier de repassage, le rythme de la conversation est ponctué par les ronflements de Coupeau, le maniement des fers à repasser, l'heure qui s'égrène lentement du clocher; les femmes repassent en entendant les sons de la rue par intervalles, car l'atelier s'ouvre au-delà de la clôture réelle de la boutique. Ici, l'efficacité tranquille de plusieurs rythmes accorde aux conditions de travail une dimension humaine. Les chants ${ }^{9}$ donnent la mesure, alors qu'à l'usine le travail est orchestré uniquement par le nombre de tours des machines.

\section{Environnement sonore des Grands Boulevards.}

Dans l'atelier, le geste au travail et les bruits sont synchrones ${ }^{10}$. Zola projette cette synchronie jusque dans les mouvements qui guident les pas dans les rues, comme dans une scène de L'Assommoir ${ }^{11}$ qui se déroule sur les Grands Boulevards. Gervaise arpente les grands cafés, où se tiennent les prostituées. Quand elle arrive près de la gare, les indications sonores sont plus précises que la description matérielle de l'espace. L'héroïne différencie les Grands Boulevards non pas à partir des délimitations visibles mais d'après des sonorités qui attirent ou bien repoussent, d'un côté à l'autre du trottoir, en fonction de la densité du piétinement des passants, du roulement des autobus et du grondement engendré par le pullulement humain.

Ces partitions sonores se distinguent entre elles. On lit par exemple qu'un fontainier va créer une "aura » particulière; plus loin, deux hommes qui discutent vivement attirent l'attention. Une identique attraction est provoquée par les sonorités qui sortent des cafés aux portes battantes, "les climats de voix qui murmurent», les voix qui «glapissent», les voix qui « gueulent», etc. Ainsi, les Grands Boulevards apparaissent plutôt diversifiés dans leur dimension sonore, moins homogènes qu'ils ne le sont visuellement. C'est une question d'heure, mais aussi de rythme, de déplacement. Les gens se pressent dans un même mouvement, celui de la foule qui mélange les populations à la sortie du travail mais les sépare dans les cafés. 


\section{Les grands magasins.}

Le XIXe siècle est celui de la construction de grands espaces clos. La rumeur qui y règne permet de rendre compte des idées d'utilitarisme de l'époque auxquelles Zola adhérait.

Dans l'air immobile, où l'étouffement du calorifère attiédissait l'odeur des étoffes, le brouhaha augmentait, fait de tous les bruits, du piétinement continu, des phrases cent fois répétées autour des comptoirs, de l'or sonnant sur le cuivre des caisses assiégées par une bousculade de portemonnaie, des paniers roulants dont les charges de paquets tombaient sans relâche dans les cuves béantes ${ }^{12}$.

Le plus souvent, l'auteur profite de cet univers phonique pour donner plus de détails sur le caractère de ses personnages. Ainsi Mouret plonge-t-il Vallagnosc en pleine foule, dans le grand magasin dont il est le directeur, "pour achever de l'étourdir, et pris lui-même du besoin physique de ce bain de succès ${ }^{13}$ ». C'est un plaisir d'entendre l'agitation autour de soi. L'activité produit une ambiance qui fait sentir le succès, qui fait croire que les affaires marchent. Dans ce processus dynamique, les sons accélèrent la sensation d'utilitarisme et donnent au directeur l'impression que la ville est en mouvement : "Au début la foule arrive : il ne se trompait plus aux bruits qui lui arrivaient du dehors, roulement de fiacres, claquement de portières, brouhaha grandissant de foule. » Au milieu de l'après-midi, c'est la cohue :

maintenant, la trépidation intérieure étouffait les bruits du dehors; on n'entendait plus ni le roulement des fiacres, ni le battement des portières ; il ne restait au-delà du grand murmure de la vente que le sentiment de Paris immense, d'une immensité qui toujours fournirait des acheteurs [...]. À la fin de l'après-midi, lentement, la foule diminuait. Des volées de cloches, à une heure d'intervalle, avaient déjà sonné les deux premières tables du soir ; la troisième allait être servie. [...] Du dehors, ne venait plus que le roulement des derniers fiacres, au milieu de la voix empâtée de Paris, un ronflement d'ogre repu ${ }^{14}$.

Que conclure au sujet de ce volume acoustique partagé ? Que la circulation et l'accélération des activités sonores entendues par les uns sont synonymes de concentration et d'étouffement pour les autres. La rumeur d'Au Bonheur des Dames est le résultat de la construction d'une "bulle de vie» en dehors de la rue. C'est l'auteur qui écrit que pour les uns «toute la vie était là, on avait tout sans sortir, l'étude, la table, le lit, le vêtement. Le Bonheur des Dames se suffisait, plaisirs et besoins, au milieu du grand 


\section{Olivier Balä̈}

Paris, occupé de ce tintamarre, de cette cité du travail qui poussait si largement dans le fumier des vieilles rues, ouvertes enfin au plein soleil ${ }^{15}{ }^{»}$. Mais pour d'autres l'ambiance nouvelle peut avoir des conséquences nocives sur leur état physique et moral, comme l'écrit ailleurs le romancier, évoquant l'ambiance sonore confuse et qui porte à l'étourdissement. Même les pièces dont « la moquette rouge étouffait le bruit des pas », où « la voix haute et lointaine du rez-de-chaussée se mourait», donnent l'impression d'une atmosphère alourdie ${ }^{16}$.

Le passage qui suit montre le confinement phonique dans l'immense espace clos qui met en réverbération les activités commerciales qu'on a l'habitude d'entendre moins confusément dans la rue :

Lhomme avait cent vingt musiciens sous sa direction, le rêve de sa vie était réalisé. Et une grande fête fut donnée dans les magasins, un concert et un bal, pour présenter la musique du bonheur à la clientèle, au monde entier. Les journaux s'en occupèrent, Bourdoncle lui-même, ravagé par ces innovations, dut s'incliner devant l'énorme réclame. Ensuite, on installa une salle de jeu pour les commis, deux billards, des tables de trictrac et d'échecs. Il y eut des cours le soir dans la maison, cours d'anglais et d'allemand, cours de grammaire, d'arithmétique, de géographie; on alla jusqu'à des leçons d'équitation et d'escrime. Une bibliothèque fut créée, dix mille volumes mis à la disposition des employés. Et l'on ajouta encore un médecin à demeure donnant des consultations gratuites, des bains, des buffets, un salon de coiffure ${ }^{17}$.

L'attachement de Zola à décrire les sons qui s'emballent, les repas bruyants, les passants qui «s'engueulent», les convergences entre les gestes et les rythmes sonores, les étourdissements, les soufflements, les roulements, les stridences, les grincements témoigne de l'intégration par ses contemporains des rythmes et des sons nouveaux, de l'acceptation par certains des bruits reflétant l'utilitarisme. Mais les scènes décrites ci-dessus racontent d'autres choses: dès que le rythme est trop rapide, dès que les sons proviennent de manière trop diversifiée au même moment, l'héroïne de L'Assommoir ne s'entend plus parler. Le tapage étouffe les cris, la désoriente. Les repères sonores de la voix eux-mêmes sont bouleversés, et on assiste à cette scène où elle appelle Goujet à mi-voix dans le bruit cadencé des marteaux et les ronflements des soufflets de la forge. On voit là déjà le Zola qui dénoncera le bruit prodigieux des machines dans Germinal (1885) :

L'idée lui était venue de tourner les robinets de décharge, pour lâcher la vapeur. Les jets partirent avec la violence des coups de feu, les cinq chaudières se vidèrent d'un souffle de tempête, sifflant dans un tel grondement de foudre que les oreilles en saignaient ${ }^{18}$. 
En insistant sur la convergence qui s'établit entre les projections des idées d'utilitarisme, liées à la conception d'un monde qui bouge, et l'accélération d'un rythme mécanique capable de fortes intensités aux fréquences sonores aiguës, Zola dit implicitement qu'au bout de ce raisonnement se trouve une ville remodelée par l'écoute. L'hérö̈ne d'Au Bonheur des Dames en est la naïve victime. Elle s'est vu attribuer par son entreprise une chambre, à laquelle elle accède par un long couloir et où elle se jette le soir après une journée de treize heures de travail. Le premier contact qu'elle a avec sa voisine, Pauline, c'est à travers la cloison. Elle l'entend se mettre au lit. Chaque soir, les mêmes bruits lui parviennent: les demoiselles qui rentrent une à une, de courtes conversations chuchotées, des rires, parfois des querelles «qu'on étouffe». Elle entend les lits craquer, les bâillements, puis le lourd silence qui s’installe; elle est effrayée par sa voisine de gauche qui «rêve souvent tout haut», par d'autres qui, malgré l'interdiction, veillent pour raccommoder, avec moult précautions pour ne pas faire de bruit. Les gestes sont ralentis, certains sont évités, «car un silence frissonnant» doit seul sortir « des portes closes ${ }^{19}$.

Alors, à travers cette atmosphère où le moindre bruit de pas venu du corridor fait lever la tête, où l'on s'entend marcher, où l'on se tait dès qu'un bruit advient, on se rend bien compte que Zola ne reconstruit pas une manière d'entendre : il décrit plutôt une adaptation auditive, celle du peuple qui se sent encadré par les bruits d'une nouvelle forme de vie dominée par les mouvements liés au travail et à la densification urbaine. Et cela s'applique jusque dans le logement.

\section{CLOISONNEMENTS ET CURIOSITÉS ACOUSTIQUES : BALZAC}

Tandis que les univers sonores de Stendhal et de Flaubert ${ }^{20}$ projettent $^{2}$ dans leurs personnages leur monde intérieur, Balzac observe les mœurs dans les maisons de rapport, les pensions, les hôtels particuliers. Il présente avec précision l'irrésistible mouvement de sensibilisation de l'oreille de ses héros curieux, voire soupçonneux. Son originalité consiste à peindre l'équilibre qui semble s'établir entre les bruits des «espèces humaines » et les espaces. Par là, l'environnement sonore et l'humanité urbaine entrent en relation spéculaire, ou, mieux, d'écho. Boulevard du Temple, c'est le théâtre. Chaussée d'Antin se concentrent, selon les moments, les mouvements «des affaires, de la mode, de l'esthétique, de la vie culturelle, du pouvoir et des caprices ${ }^{21} »$. Boulevard Saint-Germain, les cafés en terrasse sont assaillis par les êtres «modernes, soignés». Il s'agit en fait des bourgeois, les plus à même de profiter de la vie de leur époque, qui se pavanent 


\section{Olivier Balä̈}

au café devant le spectacle offert à leurs yeux et à leurs oreilles ${ }^{22}$. Marc Lancelot disait : «Il y a à Paris, et surtout dans le Faubourg Saint-Germain, une foule ennuyée qui cherche par tous les moyens de passer sa soirée dans les réunions. L'ennui en commun semble plus facile à supporter que l'ennui solitaire ${ }^{23}$. » Les rues attirent aussi du monde dès que des activités festives s'y développent ; ainsi, les Champs-Élysées le 29 juillet 1844 :

Décidément, l'illumination en valait la peine, et c'est ce que j'ai vu de plus beau, moi qui ai vu les fêtes de l'Empire !... On ne peut pas se figurer l'effet de ce palais de feu aux trois couleurs qui métamorphose la grande avenue des Champs-Élysées en une salle de bal. C'est à voir... Latéralement à la grande allée, sur le cours de la Reine, il y avait une ville, c'est le mot, de saltimbanques et de baraques pour les spectacles, qui luttaient de musique et d'annonces contre la magique illumination et le feu d'artifice ${ }^{24}$.

Le vocabulaire dit par lui-même combien Balzac aime la théâtralité urbaine, celle qui est à voir, celle qui s'écoute aussi. Dans son écriture les bruits dans les rues larges et les avenues sont parfois «effrayants", les appels «incessants » et les «luttes sonores » mémorables. À l'opposé, dans des rues étroites, chaque son semble vécu comme un événement pour les personnages qui sont là, êtres discrets dont les vies sont marquées d'abord par le succès puis par l'échec: "Hier en haut de la roue chez une Duchesse, $[\ldots]$ ce matin en bas de l'échelle, chez un escompteur ${ }^{25}$. » C'est dans ce type d'homologie entre l'espace et l'activité d'un personnage que réside tout l'intérêt trouvé à la lecture du Père Goriot. Balzac en situe l'action dans la rue "Neuve Sainte Geneviève» (aujourd'hui la rue Tournefort), entre le quartier Latin et le Faubourg Saint-Marceau. La maison est placée

à l'endroit où le terrain s'abaisse vers la rue de l'Arbalète par une pente si brusque et si rude que les chevaux la montent ou la descendent rarement. Cette circonstance est favorable au silence qui règne dans ces rues serrées entre le dôme du Val de Grâce et le dôme du Panthéon [...]. Là. les pavés sont secs, les ruisseaux n'ont ni boue ni eau, l'herbe croît le long des murs. L'homme le plus insouciant s'y attriste comme tous les passants, le bruit d'une voiture y devient un événement ${ }^{26}$.

Une semblable atmosphère se retrouve dans Eugénie Grandet, à Saumur, au bout de la rue «montueuse » qui mène au château, par le haut de la ville. Cette rue "peu fréquentée, chaude en été, froide en hiver, obscure en quelques endroits, est remarquable », nous dit Balzac, 
par la sonorité de son petit pavé caillouteux, toujours propre et sec, par l'étroitesse de sa voie tortueuse, par la paix de ses maisons qui appartiennent à la vieille ville, et que dominent les remparts [...]. Peut-être y a-t-il dans ces maisons et le silence du cloître, et l'aridité des landes, et les ossements des ruines: la vie et le mouvement y sont si tranquilles qu'un étranger les croirait inhabitées s'il ne rencontrait tout-à-coup le regard pâle et froid d'une personne immobile dont la figure monastique dépasse l'appui de la croisée, au bruit d'un pas inconnu ${ }^{27}$.

L'essentiel est là. La définition, par le son, de la forme urbaine et de la société qui l'habite et qui possède la spécificité d'être toujours «oreilles tendues» vers le voisinage. À Paris, écrit l'auteur,

où les pavés ont des oreilles, où les portes ont une langue, où les barreaux des fenêtres ont des yeux, rien n'est plus dangereux que de causer devant les portes cochères. Les derniers mots qu'on se dit là, et qui sont à la conversation ce qu'un post-scriptum est à une lettre, contiennent des indiscrétions aussi dangereuses pour ceux qui les laissent écouter que pour ceux qui les recueillent ${ }^{28}$.

\section{Face aux bruits inquiétants...}

Cette tension du corps vers les phénomènes audibles s'opère autant vers la rue depuis les fenêtres des immeubles que depuis les pièces du logement vers les autres espaces intérieurs. Par exemple, on apprend que les sons dans l'escalier de la pension Vauquer renseignent tout de suite sur le pensionnaire qui monte ou qui descend, sans qu'on ait besoin d'y aller voir. Madame la tenancière y entend «le froufrou d'une robe de soie », le «pas mignon d'une femme jeune et légère » qui file chez Goriot ${ }^{29}$. On distingue dans l'escalier, espace résonateur de l'usage et des humeurs, les comportements, les manières de faire: «Rastignac put monter à sa chambre sans faire de bruit, suivi de Christophe qui en faisait beaucoup ${ }^{30}$. »

Est-il besoin de dire que les cloisons entre les chambres, qui ne coupent jamais le contact avec le pensionnaire d'à côté, permettent d'oür autant d'indices précis? Une nuit, par exemple, Rastignac entend le père Goriot pousser un «soupir semblable à un han de Saint-Joseph »; il sort dans le couloir, approche son oil de la serrure, quand de nouveau il distingue un bruit « difficile à exprimer», et qui doit être «produit par des hommes en chaussons de lisière» montant l'escalier. Il prête l'oreille et reconnaît le «son alternatif de la respiration de deux hommes». 


\section{Olivier Balä̈}

Sans avoir entendu ni le cri de la porte, ni les pas des hommes, il vit tout-à-coup une faible lueur au second étage, chez M. Vautrin. [...] Il descendit quelques marches, se mit à écouter, et le son de l'or frappa son oreille. Bientôt la lumière fut éteinte, les deux respirations se firent entendre derechef sans que la porte eût crié. [...]

- Qui va là? cria Madame Vauquer en ouvrant la fenêtre de sa chambre. - C'est moi qui rentre, maman Vauquer, dit Vautrin de sa grosse voix. C'est singulier, Christophe avait mis le verrou, se dit Eugène en rentrant dans sa chambre. Il faut veiller pour bien savoir ce qui se passe autour de soi, dans Paris ${ }^{31}$.

Attraits et questionnements sur le voisinage marquent également la vie dans d'autres demeures balzaciennes. Plus encore, l'écoute permet de surveiller ce qui se passe autour de soi. Même les bruits très intimes en euxmêmes sont objets de curiosité: Rastignac, quand il se rend chez la comtesse de Restaud, est mal reçu par les domestiques et en est offusqué :

[il] ouvrit délibérément la porte par laquelle était sorti le valet de chambre, afin sans doute de faire croire à ces insolents valets qu'il connaissait les êtres de la maison; mais il déboucha fort étourdiment dans une pièce où se trouvaient des lampes, des buffets, un appareil à chauffer des serviettes pour le bain et qui menait à la fois dans un corridor obscur et dans un escalier dérobé. Les rires étouffés qu'il entendit dans l'antichambre mirent le comble à sa confusion ${ }^{32}$.

Reprenons soigneusement les termes avec lesquels Balzac décrit l'environnement sonore de ses personnages. Le son sec est dévolu à la rue montante et étroite; le bruit étouffé à la porte cochère, à la salle de bains. Les irruptions sonores provenant du corridor ou de l'escalier interpellent et sont l'objet d'une attention inquiète, cachée. On trouve ailleurs dans Le Père Goriot que certains bruits d'origine humaine sont capables d'encoder véritablement des relations sociales :

[Rastignac arriva] rue de Helder et demanda la Comtesse de Restaud. Avec la rage froide d'un homme sûr de triompher un jour, il reçut le coup d'œil méprisant des gens qui l'avaient vu traverser la cour à pied, sans avoir entendu le bruit d'une autre voiture à la porte. Ce coup d'œil lui fut d'autant plus sensible qu'il avait déjà compris son infériorité en entrant dans cette cour, où piaffait un beau cheval richement attelé ${ }^{33}$.

Le son vient souvent défavoriser le héros balzacien et annoncer une anxiété nouvelle. 


\section{... les résonateurs acoustiques s'imposent.}

Il semble en effet que l'aménagement intérieur de l'espace, tel que le décrit Balzac, a eu raison d'un comportement sonore qui autrefois s'exprimait sans résistance, sans douleur, en public ${ }^{34}$. L'extrait où l'on voit le cousin Pons être introduit dans le petit salon de Madame Camusot l'illustre parfaitement:

«Madame, voilà votre Monsieur Pons, et en Spencer encore! » vint dire Madeleine à la Présidente. [...] En entendant un pas d'homme dans le petit salon, qui se trouvait entre son grand salon et sa chambre à coucher, Madame Camusot regarda sa fille et haussa les épaules. "Vous me prévenez toujours avec tant d'intelligence, Madeleine, que je n'ai plus le temps de prendre un parti », dit la présidente. [...]

- Voyons, reprit-elle envoyant à sa chère Minette une figure piteuse, faut-il nous débarrasser de lui pour toujours?

- Oh, pauvre homme, répondit Mademoiselle Camusot, le priver d'un de ses dîners!

Le petit salon retentit de la fausse tousserie d'un homme qui voulait dire aussi : «je vous entends».

- Et bien, qu'il entre, dit Madame Camusot ${ }^{35}$.

Cet extrait montre que la société sonore parisienne peinte par Balzac est indissociable de la systématisation des portes, des corridors, des petits salons et autres petits boudoirs qui font l'agencement spatial de l'habitation privée. Dans celle-ci, le personnage se déplace sans faire de bruit tout en prêtant l'oreille; la cloison, la porte, le corridor ne sont pas seulement des éléments séparateurs phoniques, ce sont des résonateurs que les habitués utilisent, inaugurant un style de comportement sonore où ne pas se faire entendre permet d'écouter.

«Si vous voulez me suivre par ici, Monsieur, nous serons beaucoup mieux dans ce salon pour causer d'affaires », dit Madame Hulot en désignant une pièce voisine qui, dans l'ordonnance de l'appartement, formait un salon de jeu. Cette pièce n'était séparée que par une légère cloison du boudoir dont la croisée donnait sur le jardin, et Madame Hulot laissa Monsieur Crevel seul pendant un moment, car elle jugea nécessaire de fermer la croisée et la porte du boudoir, afin que personne ne pût y venir écouter. Elle eut même la précaution de fermer également la porte-fenêtre du grand salon, en souriant à sa fille et à sa cousine qu'elle vit établies dans un vieux kiosque au fond du jardin. Elle revint en laissant ouverte la porte du salon de jeu, afin d'entendre ouvrir celle du grand salon, si quelqu'un y entrait ${ }^{36}$. 


\section{Olivier Balä̈}

Voilà bien une confirmation que la répartition de chicanes sonores entre les différents volumes de la maison sert d'avertisseur et qu'une curiosité auditive prodigieuse semble imprégner les personnages aisés; pour un peu, on croirait qu'ils appliquent de manière prophylactique leurs oreilles aux cloisons et aux portes. Et c'est probablement chez Balzac qu'on lit avec le plus d'évidence que l'expérience acoustique au XIXe n'est pas séparable des conditions d'habitat. S'y règle le jeu subtil des répulsions et des affinités entre les individus. La curiosité s'étend dans les espaces sonores intérieurs, elle invite à tendre l'oreille au moindre mouvement dans les escaliers, aux moindres inflexions de voix des êtres proches. C'est là un effet typique du mouvement de retraite opéré par la bourgeoisie du XIX siècle, par où celleci entend définir son excellence et sa différence.

La surveillance indirecte qui fait écouter aux cloisons, aux portes, dans les escaliers n'est pas sans rapport avec d'autres traits de la vie privée bourgeoise évoqués par Balzac: l'économie du mouvement et du déplacement. Ainsi, le père Grandet « $n$ 'allait jamais chez les personnes [...], il ne faisait jamais de bruit, et semblait économiser tout, même le mouvement ${ }^{37}{ }^{\text {» }}$.

\section{Les codes sonores de l'élégance du temps.}

Que faut-il voir alors dans cette acuité sonore tournée vers l'espace intérieur? Les descriptions du ton autoritaire du père, de la douceur de la voix de la mère, du jeu ou du babil des enfants, des démarches dans l'escalier, des bruits à travers les cloisons montrent que l'oreille balzacienne se fait attentive à tout ce qui entretient la passion. Cette attention subtile aux signaux phoniques capables de dévoiler la pensée intime des sujets n'appartient qu'aux bourgeois. Rappelons l'analyse de Georges Poulet au sujet de la volonté chez les personnages de Balzac: «La volonté est comme un télescope, écrit-il. Elle supprime les distances. Elle est aussi un microphone $^{38}$.»Elle vainc la distance par le développement prodigieux de l'ouie. C'est ainsi que "grâce à la puissance avec laquelle une créature passionnée sait souvent abolir l'espace pour s'unir à son autre moi, la femme de Claës perçoit au loin le bruit des pas de son mari ${ }^{39} »$. Dans un autre passage, Georges Poulet relève cette scène où, "malgré une quadruple rangée de causeurs, et dans le bourdonnement général d'une salle de jeu, Raphaël et Valentin, par un privilège des passions qui leur donne le pouvoir d'anéantir l'espace et le temps, deviennent capables d'entendre distinctement les paroles des joueurs et de savoir qui des deux retourne le roi $^{40} »$. Quelle illustration de l'effet sonore cocktail party ${ }^{41}$ ! Car ce qui compte pour tous les personnages de Balzac, c'est de pouvoir sélectionner, de pouvoir débusquer parfois le son qui leur donnera le plus d'informations 
sur la passion de l'individu, quel que soit le bruit ambiant. Dans l'univers sonore du romancier, le terrain d'union entre les personnes n'existe que pour des individus curieux d'oü̈; mais cette curiosité est liée aux tourments que les héros ressentent: "Il faut veiller pour bien savoir ce qui se passe autour de soi, dans Paris », disait Rastignac dans Le Père Goriot. En fait, il faut veiller pour bien savoir ce qui se dit sur soi.

\section{LA CHASSE AU BRUIT, PARADE MORALE}

Dans le monde de la bourgeoisie urbaine et des gestionnaires de la ville, la moralisation du bruit, chez soi et dans l'espace public, se concrétise. Ce procès que les autorités font au bruit est un procès de civilisation. Selon les bonnes mœurs, il faut débusquer le fauteur de bruit, c'est-à-dire celui qui a un comportement sonore anormal. Il faut chasser des rues l'ambiance sonore immorale, comme on chasse les odeurs qui défient la représentation de la régulation.

L'administration de la ville du XIX ${ }^{\mathrm{e}}$ siècle sanctionne en effet les sonorités que la culture de l'époque rejette. À Lyon, les horaires de curage des fosses d'aisances sont compris entre 23 heures et 3 heures du matin ${ }^{42}$. Puisque cela se fait la nuit, les chants, cris et bruits des vidangeurs pouvant troubler le repos sont interdits ${ }^{43}$. En 1828, le curage peut commencer à 10 heures du soir et se poursuivre jusqu'à 6 heures le matin en hiver, 4 heures du matin en été ${ }^{44}$. Une ordonnance de 1838 qui interdit le curage nocturne l'été détaille les sons produits par les vidangeurs, qui tapent à grands coups pour ouvrir les guichets de leurs tonneaux, qui font claquer leurs fouets, qui chantent, qui crient, se querellent ${ }^{45}$. Cette situation dure tout le long du XIX ${ }^{\mathrm{e}}$ siècle. Le 30 janvier 1877, on autorise le curage en période diurne mais on interdit toujours le claquement de fouet et autres accents vocaux qui forment le langage du métier: "Les manœuvres de service seront commandées à l'aide d'un sifflet. » Déjà, le 16 mai 1874, le règlement du service des vidanges ${ }^{46}$ indique : "Les vidangeurs devront se parler à voix basse et les manœuvres du service se feront à l'aide d'un sifflet. » De telles remarques, isolées dans la masse des ordonnances municipales $^{47}$, permettent de percevoir la pression diffuse des signaux sonores qu'on impose pour régler les comportements des agents mis au service de la collectivité dans les rues.

Les élites prônent aussi la vigilance acoustique dans la demeure. Sans la présence de bruits habituels auxquels elles s’identifient, le logis n'est guère habitable - à condition toutefois que les contacts sonores immédiats et 


\section{Olivier Balä̈}

intempestifs soient apaisés. Le désir d'un coin à soi et la solitude semblent préparer une acuité auditive nouvelle. Michelle Perrot écrit : «Distribution et usage des pièces, escaliers et couloirs de circulation des personnes et des choses, lieux de retraite, des soins et des plaisirs du corps et de l'âme obéissent à des stratégies de rencontre et d'évitement que traversent le désir et le souci de soi. Cris et chuchotements, rires et sanglots étouffés, murmures, bruits de pas que l'on guette, portes qui grincent, pendule impitoyable tissent les ondes sonores de la maison ${ }^{48}$. $\gg$ Dans des espaces d'appartement immenses et débarrassés des voix humaines, l'oreille proustienne guette dans le silence des résonances à l'état de solitude: "Je me rendormais, et parfois je n'avais plus que de courts réveils d'un instant, le temps d'entendre les craquements organiques des boiseries ${ }^{49}$. » Louis Chevalier (1958) cite Delphine Gay, qui parle, à propos des appartements en ville, « des soupirs de la cheminée $\mathrm{e}^{50}$ ».

L'expérience sonore individuelle révèle de tendres souvenirs et la littérature est riche en ce domaine. C'est Proust encore qui remarque qu'entre deux salles de bains l'écoute de l'intimité qui est souvent permise à l'hôtel du fait du rapprochement des pièces est « si rare » à Paris ${ }^{51}$, compte tenu de la grandeur des appartements. Dans le même temps, la quête d'un environnement sonore où l'on puisse s'isoler s'affirme et prépare une acuité auditive comparable à celle d'aujourd'hui, acuité pas très éloignée de celle, déjà évoquée, des personnages que Balzac décrivait sur les Grands Boulevards.

Pourtant, à la lecture de ces deux auteurs forts de la littérature (et les textes de Flaubert, Stendhal et Proust le confirment aussi), on voit que la réciprocité des échanges acoustiques entre les personnages reste le plus souvent de mise. En somme, l'espace sonore, dedans comme dehors, se compose de rythmes rarement hermétiques les uns par rapport aux autres ; les personnages, pour l'essentiel, partagent le même espace sensible mais chaque culture lui donne une écoute particulière. Ainsi, au XIX ${ }^{\mathrm{e}}$ siècle on a rencontré des personnages qui tentaient de contrôler des sons porteurs de sens pour eux-mêmes. On a pu reconnaître le développement de discours concernant l'art d'utiliser l'antichambre, ou encore constater l'existence de franges, de limites pouvant régler les sons en fonction de l'attente de l'individu. Pour sa part, Zola a repéré que la production sonore humaine peut donner matière à la ville, matière qui a parfois pour fonction d'entretenir un bouillonnement constructif, des expressions nouvelles sur le mode de vie. Pour Balzac, les préoccupations acoustiques participent d'un nouvel esprit de l'appartement, où la différenciation des espaces et la définition de l'usage social des entités du logement sont contemporaines de la constitution d'une sphère privée et de la valorisation de la famille nucléaire au sein du domicile: assignation des femmes à la sphère domestique, reconnaissance d'un statut propre à l'enfant... La prise en compte d'espaces sonores 
différenciés s’inscrit dans ce cadre: les gens instruits et aisés recommandent pour leur maison certaines formes du parler, une certaine retenue au moment du repos ou de la visite de gens extérieurs (salle à manger et cuisine ne doivent pas communiquer, chambre à coucher et pièces de réception doivent être séparées). Ces personnes établissent de nouvelles temporalités pour les activités dans l'espace et le temps de la domesticité. À l'acoustique, ils prêtent les plus hautes valeurs, et pour préserver l'intimité, et pour pouvoir entendre sans être vu.

Dès lors, les propositions architecturales (telles qu'elles apparaissent dans les traités et les projets) traduisent moins la volonté de réduire le bruit que celle de contrôler la circulation des sons ${ }^{52}$. Ainsi, il n'y est pas seulement question de l'isolation phonique, mais d'atmosphères acoustiques dans les grands magasins, les gares, les usines, les habitats bourgeois, qui assurent des repères, qui permettent de se sentir lié à son environnement culturel, d'être en cohérence avec son habitat et son réseau sonore de socialité : une porte laissée ouverte permet une surveillance subtile, discrète et efficace; un grand magasin permet de déambuler à distance des autres tellement la rumeur est vivace.

Ces descriptions sonores du passé montrent qu'au XIX ${ }^{\mathrm{e}}$ siècle on s'affranchit des principes d'isolation phonique et on parle de la dimension sonore comme d'une dimension du désir, de la création, et non de la répression.

Olivier BALAŸ

olivier.balay@lyon.archi.fr

Architecte, urbaniste, enseignant ENSAL CRESSON, GNRS / BRAUP / ENSA, Grenoble

NOTES

1. Y. Lequin, "Les citadins et leur vie quotidienne», in G. Duby (dir.), Histoire de la France urbaine, t. 4, La Ville de l'âge industriel, Paris, Seuil, 1983, p. 354.

2. Sur les processus de déculturation au XIX ${ }^{e}$ siècle, cf. M. Crubellier, "Les citadins et leurs cultures », in ibid., p. $360 \mathrm{sq}$. Crubellier rappelle cette définition de la déculturation donnée par Jules Simon, un philanthrope, ministre sous la Troisième République : «L'ouvrier vit comme un paria, au milieu d'une civilisation inconnue et d'un courant d'idées qui ne descendent pas jusqu’à lui; il n'est pour ainsi dire ni de son pays ni de son temps; privé de toutes les joies intellectuelles, il se rejette, comme la brute, sur des plaisirs grossiers qui achèvent de le dégrader» (p. 364).

3. Cf. O. Balaÿ, L'Espace sonore de la ville au XIX siècle. Une face cachée de l'art de construire, Bernin, À la Croisée, 2003.

4. E. Zola, L'Assommoir (1877), Paris, Le Livre de Poche, 1974.

5. Ibid., p. 460. 


\section{Olivier Balä̈}

6. Ibid., p. 461-462.

7. Ibid.

8. Ibid., p. 190-191.

9. Cf. à ce sujet L. Mumford, Technique et Civilisation, traduction de D. Moutonnier, Paris. Seuil, 1978, p. 185.

10. Indiquons que ces deux descriptions mettent en comparaison les relations qui existent entre le travailleur manuel et son bruit. La scène dans l'usine où les deux forgerons cherchent à séduire Gervaise par la «danse» de leurs coups portés sur l'enclume montre combien la mise en rythme du corps au travail est liée à la répétition sonore automatique produite par les machines environnantes. Zola indique au lecteur de son époque un phénomène qu’on peut vérifier aujourd'hui, à savoir l'adaptation d'un rythme personnel à celui du bruit ambiant, exprimant d'une certaine façon une possibilité de communication interindividuelle. Cf. E. T. Hall, Le Langage silencieux, Paris, Seuil, 1984; et la synthèse proposée par Jean-Paul Thibaud sur cette question : La Notion de synchronie sonore dans les communications humaines, mémoire CEAA Architecture et environnement sonore, École d'architecture de Grenoble, CRESSON, 1988.

11. E. Zola, L'Assommoir, op. cit., fin du chapitre 12.

12. E. Zola, Au Bonheur des Dames (1883), Paris, Le Livre de Poche, 1976, chap. 4, p. 130.

13. Ibid.

14. Ibid., p. 119,130 et 139.

15. Ibid.

16. Ibid., p. 133.

17. E. Zola, Au Bonheur des Dames, cité in catalogue Les Expositions de Paris, G. Davioud architecte de Paris, Paris, 1981.

18. Cité par R. M. Schafer, Le Paysage sonore, Paris, JC Lattès, 1979, p. 113.

19. E. Zola, Au Bonheur des Dames, op. cit., p. 151-154.

20. O. Balaÿ, L'Espace sonore de la ville au XIXe siècle, op. cit.

21. F. Paul-Lévy, La Ville en croix: de la révolution de 1848 à la rénovation haussmannienne. Éléments pour une problématique générale, Paris, Librairie des Méridiens, 1984, p. 62.

22. R. Sennett, Les Tyrannies de l'intimité, Paris, Seuil, 1979, rééd. 1995, p. 173. Cf. aussi F. Paul-Lévy, La Ville en croix, op. cit., chap. 3, p. 71-73.

23. Cité par T. Zeldin, Histoire des passions françaises, t. 5, Anxiété et Hypocrisie, Paris, Encres, 1979, p. 106.

24. H. de Balzac, lettre à Mme Hanska du 29 juillet 1844, Correspondance, Paris, Gallimard, «Bibl. de la Pléiade», 2006.

25. H. de Balzac, Le Père Goriot, La Comédie humaine, t. II, Paris, Gallimard, «Bibl. de la Pléiade», 1935, p. 884.

26. Ibid., chap. 1. L'action a lieu en 1819 à Paris.

27. H. de Balzac, Eugénie Grandet (1933), La Comédie humaine, t. III, Paris, Gallimard. «Bibl. de la Pléiade», 1935, chap. 1. L'action a lieu entre 1819 et 1833.

28. H. de Balzac, Le Cousin Pons (1847), Paris, Le Livre de Poche, 1972, p. 117.

29. H. de Balzac, Le Père Goriot, Paris, Le Livre de Poche, 1972, p. 34.

30. Ibid., p. 42.

31. Ibid., p. 66.

32. Ibid., p. 68.

33. Ibid., p. 69.

34. R. Sennett, Les Tyrannies de l'intimité, op. cit., p. 273 sq.

35. H. de Balzac, Le Cousin Pons (1847), La Comédie humaine, t. VI, Paris, Gallimard. «Bibl. de la Pléiade», 1936.

36. H. de Balzac, La Cousine Bette (1847), Paris, Gallimard, «Folio», 1972, p. 34. On trouvera dans ce roman une description rapide du quartier du Louvre au XIXe siècle.

37. H. de Balzac, Eugénie Grandet, op. cit., chap. 1.

38. G. Poulet, Étude sur le temps humain. La distance intérieure, Paris, Plon, 1952.

39. Ibid., p. 151.

40. Ibid. 


\section{Stridences et chuchotements}

41. L'effet sonore «cocktail party» désigne l'aptitude à discerner une voix parmi beaucoup d'autres, à ignorer une quinte de toux de son voisin lorsqu'on écoute de la musique dans une grande salle, etc. Cf. J. R. Pierce, Le Son musical, Paris, Belin, «Pour la science», 1984.

Effet voisin, l'effet sonore de «créneau»: «L'auditeur peut alors continuer à discriminer le message sonore qui lui est destiné, bien que celui-ci ne ressorte pas de façon particulière par rapport au bruit ambiant»(J.J. Deletre, Répertoire des effets sonores, cinq échantillons, Grenoble, CRESSON, février 1987).

42. A.M., collection reliée des ordonnances de police non classées depuis 1806 jusqu'à 1849 . ordonnance du $1^{\mathrm{er}}$ janvier 1823 (volume 1806-1830).

43. Ibid.

44. Ibid., ordonnance du 15 décembre 1828 (volume 1835).

45. Ibid., ordonnance du 26 septembre 1838 (volume 1831-1849).

46. A.M., série I1.129 (après 1870) : police urbaine, nettoiement, vidange, égouts, ordonnances du 16 mai 1874 et du 30 janvier 1877 .

47. Un exemple: tous les habitants de la ville (ordonnance du 17 janvier 1806) sont tenus de balayer ou de faire balayer, chaque jour, exactement avant 7 heures du matin en été et avant 8 heures en hiver, les rues dans toute l'étendue qu'ils occupent; ils doivent en outre faire arroser le pavé chaque jour en été à 9 heures et à 14 heures. L'entrepreneur du nettoiement fera passer chaque matin un préposé qui, au son de la cloche, annoncera le passage prochain des tombereaux (ordonnance du 15 décembre 1811); ainsi, le tombereau no 20 passera

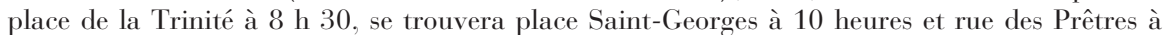
11 heures.

48. M. Perrot, "Manières d'habiter », in Ph. Ariès et G. Duby, Histoire de la vie privée, t. 4, De la Révolution à la Grande Guerre, Paris, Seuil, 1987, p. 310.

49. M. Proust, "Combray», Du côté de chez Swann, À la recherche du temps perdu, Paris, Gallimard, 1954.

50. In A. Corbin, Le Miasme et la Jonquille. L'odorat et l'imaginaire social, XVIII ${ }^{e}$ XIXe siècles, Paris, Aubier-Montaigne, 1982, p. 191.

51. M. Proust, La Prisonnière, Paris, Gallimard, «Folio», 1954, p. 10.

52. Cf. O. Balaÿ, L'Espace sonore de la ville au XIXe siècle, op. cit. 